\title{
Research of Neighbor Discovery for IPv6 over Low- Power Wireless Personal Area Networks
}

\author{
Bingqing Luo; Suning Tang; Zhixin Sun \\ Key Laboratory of Broadband Wireless Communication and Sensor Network Technology \\ Nanjing University of Posts and Telecommunications, \\ Nanjing, China \\ Email: sunzx@njupt.edu.cn
}

\begin{abstract}
The Ipv6 neighbor discovery protocol is unable to meet the networking and address configuration requirements of the nodes in the wireless sensor network (WSN). To address this problem, the 6lowpan network architecture is presented in this paper, and based on the architecture, a method for configuring addresses of the 6lowpan nodes and a basic process for interaction during neighbor discovery are proposed. A context management and distributing strategy is also proposed to expanded 6lowpan domain, providing an approach to the standard protocol RFC6775. Simulation results show that the proposed 6lowpan neighbor discovery protocol is highly feasible and effective.
\end{abstract}

Keywords-6LoWPAN; neighbor discovery; context; header compression; address configuration

\section{INTRODUCTION}

The IPv6 in Low-Power Wireless Personal Area Networks (6LoWPAN) [1] is an IPv6-based low-speed wireless personal area network standard. The 6LoWPAN Working Group was created by the Internet Engineering Task Force (IETF) Working Groups to standardize an adaptation layer on top of IEEE 802.15.4 [2] physical (PHY) layer for IPv6 network [3]. The adaptation layer provides header compression and packet fragmentation/reassembly, enabling IPv6 packets to be carried on the WSN.

The 6LoWPAN Neighbor Discovery (6LoWPAN-nd) protocol is an indispensable part of the 6LoWPAN standard. It specifies the node address configuration methods, networking process and the neighboring route discovery process. But the standard IPv6 neighbor discovery protocol is unsuited for lowpower wireless network and cannot support the operation of the adaptation layer in 6LoWPAN. Therefore, many works have been done to simplify the IPv6 neighbor discovery protocol and incorporate necessary functions $[4,5]$. IETF released the latest neighbor discovery protocol RFC6775 [6] in 2012. However, the 6lowpan-nd protocol proposed by Shelby et al. failed to provide detailed schemes for command overhead control, node registration, node acknowledgement and the support for the 6LoWPAN wireless sensor network with multiple border routers.

In addition to the 6LoWPAN network architecture, this paper proposes a neighbor discovery protocol for 6LoWPAN, including the design of the address configuration method, the basic protocol for 6LoWPAN neighbor discovery and interaction, and the enhanced context distribution and synchronization strategy for the expanded 6LoWAPN domain. Finally, the 6LoWPAN and neighbor discovery protocols in the kernel of Contiki are improved via Cooja, achieving communication and networking between the 6LoWPAN node and the gateway. The test demonstrates the feasibility and effectiveness of the proposed neighbor discovery protocol.

\section{Design OF THE 6LoWPAN NeTWORK ARChitecture}

Fig.1 shows the 6LoWPAN network architecture consisting of sensor nodes, routers, 6LoWPAN border routers (6LBRs), resource management platform and several Internet of Things applications. On the sensing layer, the architecture classifies the device nodes with heterogeneous codes or protocols using the virtual domain classification method. Unlike what the Internet gateway means, 6LBRs is on the network layer, meaning that it needs to forward the packets on the network layer and adapt to different sensing networks by performing address configuration and conversion. Due to the diversity, heterogeneity and large number of terminals in the Internet of Things environment, the Internet of Things resource management platform locates and acquires resources effectively via terminal resource addrress.

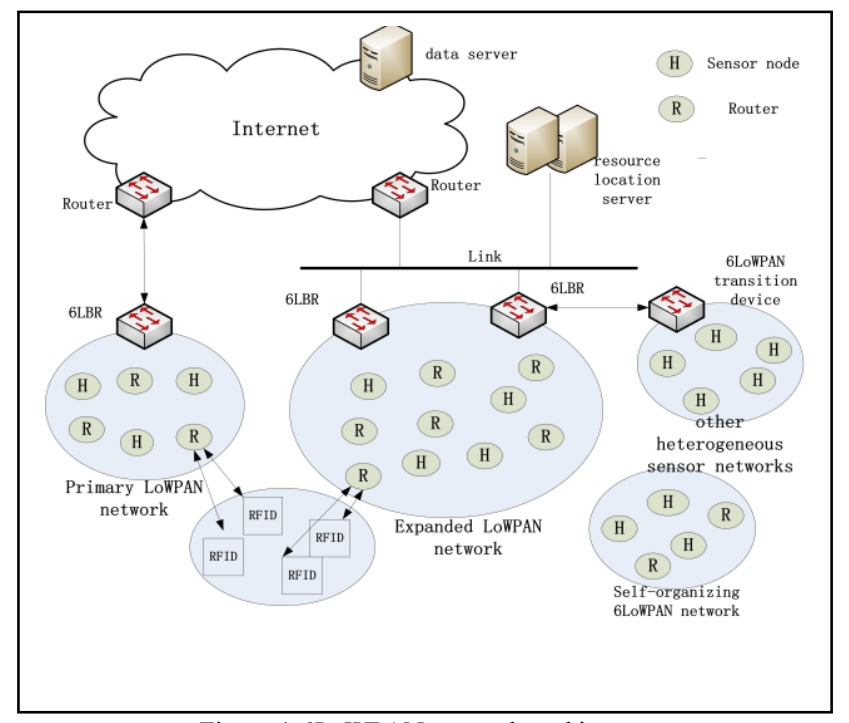

Figure 1 6LoWPAN network architecture 
Definition 1 6LBR: the gateway device directly connecting 6LoWPAN with other networks. It is also responsible for forwarding packets, managing and configuring node addresses in 6LoWPAN.

Definition 2 6LoWPAN node /Router(LN/LR): the node capable of running the 6LoWPANprotocol stack. Each LoWPAN node has a unique IPv6 address, is capable of sending and receiving IPv6 packets, and supports ICMPv6 and UDP.

Definition 3 6LoWPAN network: the wireless sensor network consisting of $\mathrm{n}$ 6LoWPAN nodes. Each domainis comprised of 6LoWPAN nodes sharing the same IPv6 address prefix (first 64 bits). The node's IP address remains, regardless of its position in the area.

Definition 4 Primary LoWPAN network: the LoWPAN domain by which the sensor nodes connect with the Internet via an Internet of Things gateway.

Definition 5 Expanded LoWPAN network: the LoWPAN domain by which the sensor nodes connect with the Internet via several Internet of Things gateways.

\section{DESIGN OF THE 6LOWPAN BASED NeIGHBOR DISCOVERY PROTOCOL}

\section{A. Address configuration scheme}

6LBRs configures addresses (e.g. 16-bit short address and IPv6 address) of nodes in 6LoWPAN using the address configuration scheme. The greatest characteristics of 6LoWPAN is that all network nodes use the IPv6 address format. The 6LoWPAN address compression protocol supports many IPv6 addressing methods, such as the address generated by adding a prefix to IID from the local link address, Dynamic Host Configuration Protocol (DHCP), and the multicast address.

Due to its ability of stateless auto-configuration of address, IPv6 is more suited for unsupervised and data-intensive Internet of Things scenarios. An IPv6 address can be allocated to each node without the use of external servers and the need to manually configure nodes. Therefore, 6LoWPANuses IPv6's stateless auto-configuration of address to generate the IPv6 address via the link layer address.

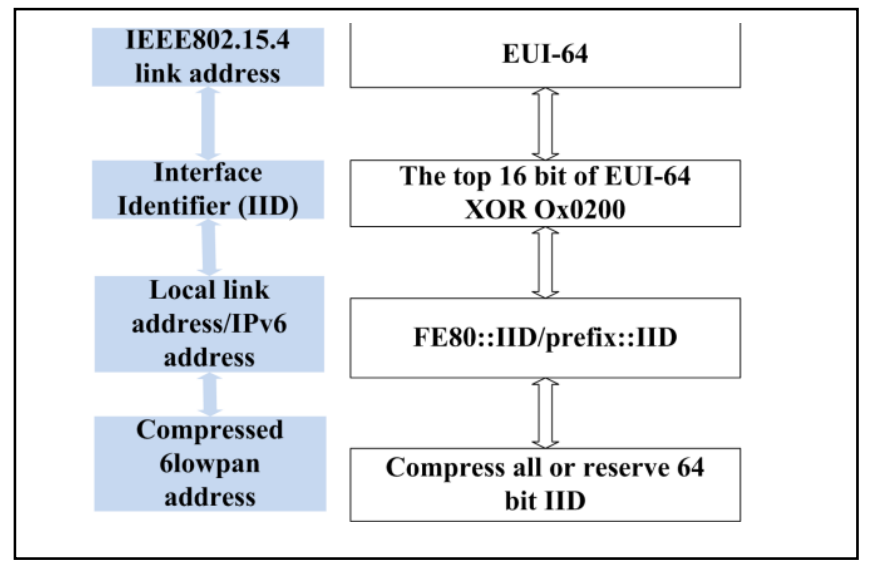

Figure 2 IPv6 address configuration

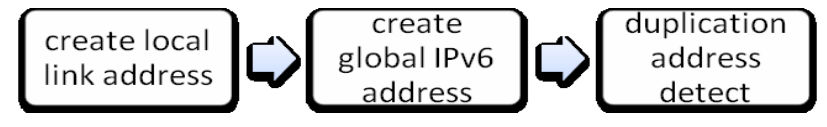

Figure 3 IPv6 address generation process

To achieve cross-network global routing, the address field can be totally omitted after the IPv6 address derived from the globally unique link layer address is compressed using the 6LoWPAN protocol. Hence, the 6LoWPAN node can generate the IPv6 address via the internationally unique device link address (i.e. EUI-64 address), i.e. prefix + EUI-64. Fig.2 shows how the EUI-64 address forms the IPv6 address and the address format after being compressed via the 6LoWPAN protocol.

Fig. 2 shows the 6LoWPAN network addressing method. A complete addressing system still requires a series of address generating processes and address registration schemes, as shown in fig.3. First, 6LoWPAN nodes generate the local link address using their own EUI-64 address. The network prefix can be acquired via interaction between the local link address and 6LBR. The global IPv6 address is configured using the prefix, and the configured address is then registered with 6LBR. The code addressing process is completed after 6LBR perform srepetitious address detection. The steps above are all based on the 6LoWPAN neighbor discovery protocol.

Meanwhile, the prefix of the context used for compressing the 6LoWPAN address is distributed by the neighbor discovery protocol. The scheme for context management and distribution is discussed in detail in Section C.

\section{B. The basic protocol of 6LoWPAN-ND}

Consider the low-power low-speed properties of the wireless sensor network, the Neighbor Discovery Protocol (Ipv6 ND) [7] is simplified in this paper. To achieve this, the functions regarding the Neighbor Solicitation message multicasting in the IPv6 ND protocol, the periodic reception of the Router Advertisement (RA) message, and the address analysis by the nodes, are omitted. The complicated schemes are carried out by the boundary router as much as possible. Basic ND message and options are retained, such as Router Solicitation (RS),Router Advertisement(RA), Neighbor Solicitation(NS), Neighbor Advertisement(NA), Source link layer address option (SLLAO), and Prefix information option(PIO). Some new options are added for the 6LoWPAN protocol, such as 6LoWPAN Context Option (6CO), authorized border router option(ABRO) and the address registration option. The fundamental functions of the basic messages and options used in 6LoWPAN-ND are listed in TABLEI, where the red options are the newly added options to the IPv6 ND protocol.

\begin{tabular}{|c|c|c|c|c|}
\multicolumn{5}{|c|}{ TABLEI BASIC MESSAGES AND OPTIONS IN 6LOWPAN-ND } \\
\hline $\begin{array}{c}\text { ND } \\
\text { messages }\end{array}$ & $\begin{array}{c}\text { Source } \\
\text { address }\end{array}$ & $\begin{array}{c}\text { Destination } \\
\text { address }\end{array}$ & Options & Notes \\
\hline RS & LLA(A) & $\begin{array}{c}\text { All-router } \\
\text { multicasting } \\
\text { address or } \\
\text { LLA(B) }\end{array}$ & SLLAO & $\begin{array}{c}\text { Local address of } \\
\text { thelink carrying A }\end{array}$ \\
\hline RA & LLA(B) & LLA(A) & SLLAO & $\begin{array}{c}\text { The SLLAO option } \\
\text { in the RS message }\end{array}$ \\
\cline { 3 - 5 } & & ABRO & $\begin{array}{c}\text { Carry the } \\
\text { authorization } \\
\text { message of the }\end{array}$ \\
\hline
\end{tabular}




\begin{tabular}{|c|c|c|c|c|}
\hline & & & PIO & $\begin{array}{c}\text { The prefix of the } \\
\text { local network }\end{array}$ \\
\cline { 4 - 5 } & & & 6 CO & $\begin{array}{c}\text { The prefix and the } \\
\text { corresponding } \\
\text { context ID of the } \\
\text { local network or the } \\
\text { network where the } \\
\text { host C is located }\end{array}$ \\
\hline NS & LLA(A) & LLA(B) & SLLAO & $\begin{array}{c}\text { Local address of } \\
\text { thelink carrying A }\end{array}$ \\
\cline { 3 - 5 } & & & ARO & $\begin{array}{c}\text { The temporary } \\
\text { global IPv6 address } \\
\text { carrying A registers } \\
\text { with B }\end{array}$ \\
\hline NA & LLA(B) & LLA(A) & ARO & $\begin{array}{c}\text { The global IPv6 } \\
\text { address carrying A } \\
\text { and its registration } \\
\text { status }\end{array}$ \\
\hline
\end{tabular}

As mentioned above, when newly added to the network, the 6LoWPAN node makes the router request, acquires the prefix (prefix,context)and registers the address via the neighbor discovery protocol. The prefix and address information is periodically maintained and updated in subsequent communication processes. Fig. 4 shows the basic interaction process for discovering the neighbor in 6LoWPAN.

During the network initialization, the address of the newly added node has not been configured and registered, so the 6LoWPAN packets transmitted within the network are labelled with the local address of the link. In this case, the format of the data transmitted in the 6LoWPAN network is shown in fig.5.

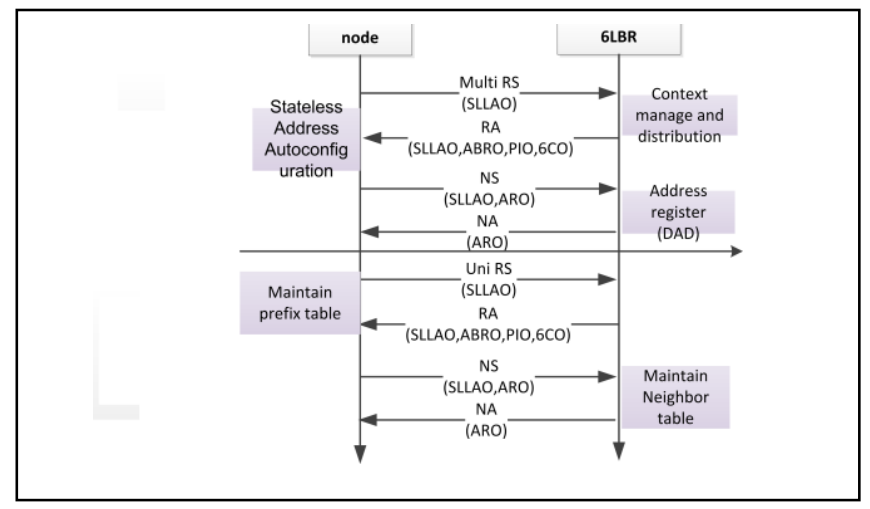

Figure 4 Basic interaction scheme in 6LoWPAN-ND

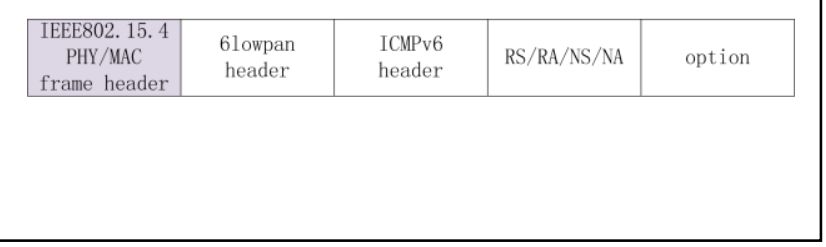

Figure 5 Data format during network initialization
Because 6LoWPAN-ND is a simplified version of IPv6 ND, we briefly describe the interaction steps and the new options it carries. The network initialization and subsequent communication processes share almost the same interaction steps, the following discussions are focused on their difference.

Step 1: Node (A) generates the local link address via the link layer EUI-64, and the SLLAO option is formed for interaction in the neighbor discovery protocol.

Step 2: A acquires the router and its prefix information by sending the multicast RS message carrying the SLLAO option. A default router and its network prefix are chosen. SLLAO carries the local link address of the sensor node.

During network initialization, A chooses a default router by sending the multicast RS message. But in the subsequent communication process, A acquires the router and prefix information for its router again directly by sending the unicast RS message. The sending of this unicast message is only triggered when the valid lifetime of the default router or its prefix (prefix,context) is approaching.

Step 3: On reception of the RS message, 6LBR(B) duplicates its SLLAO option so that it can be carried when replying to the RA message.

Step 4: The unicast RA message is sent to the node A via the RA message. In addition to the SLLAO option, it also carries the context option $6 \mathrm{CO}$ and the authoritative border router option (ABRO).

The node A only receives the prefix and context that is not only consistent with the default ABRO message but also sent by the border router. Prefix denotes the prefix of the 6LoWPAN domain and is carried by the PIO option. The context message carries the prefix for address compression. If the IPv6 host (C) is from the external network, then the RA message may carry the prefix (i.e. context(c)) of the network where the host $\mathrm{C}$ is located. Therefore, a RA message can carry several 6CO options simultaneously.

Step 5: The node A generates a temporal global unique IPv6 address IP(A) in a prefix+IID manner. Only on reception of the acknowledgement from the gateway will it be confirmed as the formal IPv6 address.

Step 6: A registers the address by sending the ARO option in the NS message to the gateway B, and ARO carries the temporal IPv6 address IP(A). During subsequent communications, A periodically sends the NS message carrying the ARO option to B.

Step 7: On reception of the NS message from A, the gateway $\mathrm{B}$ records $\mathrm{IP}(\mathrm{A})$ stored in its $\mathrm{ARO}$ and queries its neighbor's caching table. If the table is not full and no address items are the same in the table, add a new item to the neighbor's caching table and makes a reply to the successfully registered ARO option via the NA message. Otherwise, make a reply to the ARO option that fails in registration.

\section{Context management and distribution scheme}




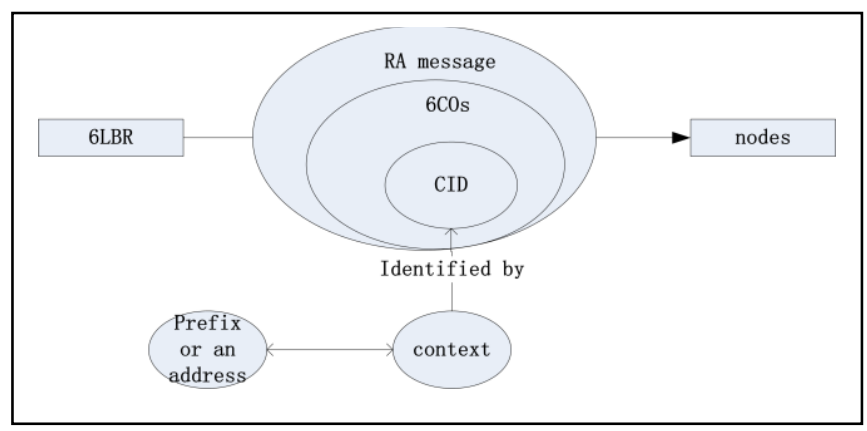

Figure 6 The concept about context

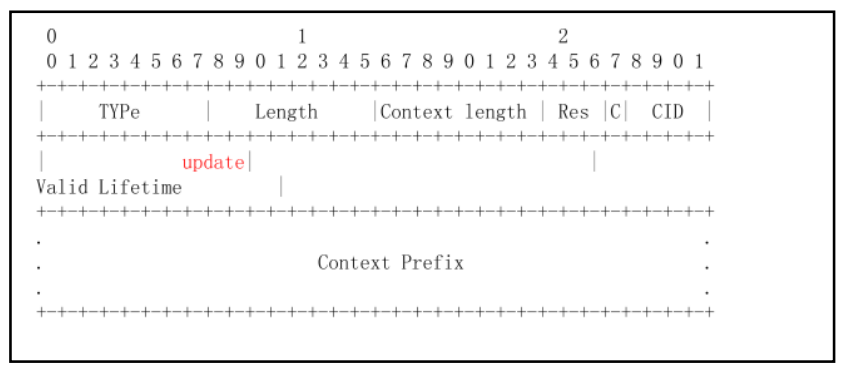

Figure 7 6LoWPAN Context Option format

If the context-based compression methodis chosen for the packets in the lowpan domain, then 6LBR in the lowpan domain needs to configure and manage the context and the context ID. If the context-based compression methodis chosen for the packets in the expanded lowpan domain, then the context information in two or more border routers is required to be consistent with each other.

The context is an IPv6 prefix of any length or address (/128). Context information is carried in optional 6CO, which allows dissemination of multiple contexts identified by a Context ID(CID). Fig.6 presents the 6LoWPAN Context Option format, where C, CID, Valid Lifetime and Context Prefix are configured by $6 \mathrm{LBR}$. Up to $166 \mathrm{COs}$ are carried in RA message [6]. Usually, it is the 6LBR to manage the context entry and disseminate them to nodes through RA message. When a $6 \mathrm{CO}$ option is received in a RA message by nodes, it can be employed to add or update the information in Context Table (CT), a conceptual data structure maintained by sensor nodes and routers for the context information received from 6LBR. The whole concept of the context is shown in fig.7.

\section{1) Multi-source synchronization strategy in the expanded 6lowpan domain}

Based on the context synchronization within the 6lowpan domain with a single border route in the neighbor discovery protocol, a multi-source synchronization strategy is introduced in this paper to enable the information and time synchronization of the context between different border routes. That is, the context information of the border routes is first synchronized and then the border routes disseminate this context information so that the context information and version numbers received by the routes and hosts in the expanded lowpanare from the same virtual border route. This provides an approach to the synchronization of contexts from different border routes in the expanded lowpan domain.
According to RFC6775, the original prefix information in the border gateway is configured manually or acquired through the DHCPv6 protocol. Therefore, we should first ensure the consistency of the prefix information (i.e. the consistency of the context table created by 6LBRS) when 6LBRS is originally configured (unless stated otherwise, 6LBRs hereafter refers to the border routes in the same expanded 6lowpan domain). The consistency information that needs to be checked in PIO is as specified as in the RFC4861 standard literature; the consistency information that needs to be checked in $6 \mathrm{CO}$ includes the context prefix, valid lifetime and CID. Afterwards, the version number in the ABRO option created by each LBR is updated and the multi-source synchronization between 6LBRs is triggered. The updating of the version number is related to the prefix content, its valid lifetime, $\mathrm{CID}$ in $6 \mathrm{CO}$ and its valid lifetime.

The context updating scenarios that may occur in the 6lowpan domain can be classified into the following categories: (a) a 6LBR acquires the IPv6 address prefix of a communicating host in the external network by using the DHCPv6 protocol, adds its address prefix to the context table items, updates the version number of this table item, and triggers a new round of multi-source synchronization. (b) The valid lifetime of a context expires and is equal to 0 , and the table item needs to be deleted. (c) The valid lifetime of a context is changed. All these changes can trigger the multisource synchronization scheme, and the synchronization steps are as follows:

STEP 1:Set the update option in the 6CO table item to 1, meaning that this table item enters the updating and synchronization stage, unlike the reply to the RS message and the distribution of the periodic RA message.

STEP 2: This 6LBR will broadcast the updated CID, context content and the version number (i.e. the $6 \mathrm{CO}$ item, including the update label), to other 6LBRs and wait for their replies.

STEP 3: On reception of the 6CO items whose update is set to 1 , other border routes compare the received version number and the local version number. If the received version number is larger than the local one, then it will update the CID record, store the new version number, set update to 0 , and reply to the response message. If the received version number is less than or equal to the local one, then it will not perform the updating operations, abandon this option and send the acknowledgement.

STEP 4: On reception of all LBRs' acknowledgements, this $6 \mathrm{LBR}$ will set update among the $6 \mathrm{CO}$ items to 0 , and write the new prefix context information into the $6 \mathrm{CO}$ items.

For border routes that initiate multi-source synchronization, setting of the Update flag bit can prevent the routes from distributing the context items to routes and nodes within the 6lowpan domain before finishing synchronization with other border routes. For other synchronized border routes, it can effectively determine the updating information is the synchronization information from between border routes or from other synchronization information. 
2) Method for distributing the context information within the expanded 6lowpan domain

The synchronization strategy above only guarantees the uniqueness of the context items configured between6LBRs and the version numbers in the ABRO options. But while distributing the items, there are so many border routes and there exists the item synchronization problem.

The border routes distributes the context information by distributing the RA messages. According to the standard, there are two scenarios for the RA message: (a) the border routes periodically distribute the context information while periodically distributing the RA message; (b)the host node sends the RS request and the border route acknowledges the RA message.

\section{a) The method of 6LBRs periodically distributing the context information}

While distributing the context information within the 6lowpan domain, two time intervals can be set: MinRtrAdvInterval and MaxRtrAdvInterval. The periodic distribution interval is $600 \mathrm{~s}$ at most and $0.33 *$ MaxRtrAdvInterval by default. According to this standard, the concept of synchronization time is introduced in this paper. That is, before periodically distributing the RA message, all 6LBRs wait for a period of synchronization time before periodical distribution. Setting of the synchronization time is helpful for border routes in ensuring the consistency of the context information between border routes before periodically distributing the RA message. The duration of the synchronization time can be determined based on the configurable maximum and minimum periodic distribution intervals. Therefore, some scope for the adjustment of the synchronization time should be allowed by considering the network conditions and the number of nodes in the 6lowpan domain. The reference value of the synchronization time is set to $0.05^{*}$ MaxRtrAdvInterval (MaxRtrAdvInterval $>600 \mathrm{~s}$ ) in this paper. If MaxRtrAdvInterval $<=600 \mathrm{~s}$, then the default value is 30 s.

During the synchronization time, 6LBRs have three states: in the first state, there is no new update to trigger synchronization, so wait until the end of the synchronization time to trigger a $6 \mathrm{LBR}$ to send the synchronization information; on reception of all acknowledgements from 6LBRs, distribute the RA message to other LR and Host. In the second state, an update that can trigger synchronization is created during the synchronization time,so wait until the end of synchronization to enter the stage of RA message distribution. In the third state, an update is ongoing during the synchronization time, so wait until the end of synchronization to enter the stage of RA message distribution. The method for periodically distributing the context relies on the multi-hop RA message distribution approach in Neighbor Discovery Optimization for 6LoWPAN draft-ietf-6lowpan-nd-18. The interaction processing between LRs or between LR and LNs, as well as the RA receiving and forwarding process are all the same as the single-LBR6lowpan domain processing steps.

The design above can ensure that all the context information received by hosts in the expanded 6lowpan domain is the version of the context information that has been synchronized by 6LBRs. This guarantees the consistency of the context information of the hosts in the domain, enabling hosts to normally compress and decompress addresses of the 6lowpan packets.

b) LN sends a RS request to LBR to update the context because the valid lifetime of the context item expires

Change of any context item can promptly trigger the item synchronization between 6LBRs. The synchronization time that lasts before periodic distribution of the RA message guarantees updating and further ensures synchronization of the context items between 6LBRs. Therefore, when a LR sends the RS request, to prevent the host from multicasting RS due to the failure to promptly acknowledge the RA message, 6LBR that receives the RS request directly sends the context information of the locally available version to the host to update the context information in the host.

\section{EXPERIMENTS}

The Cooja simulator is used in this paper to achieve networking and communication between 6LoWPAN sensor nodes and 6LBR nodes by modifying the 6Lowpan and neighbor discovery protocols in the contiki system. The effectiveness and feasibility of the proposed protocol is demonstrated.

The packets captured by tap0 during initialization is shown in fig.8. It can be seen that during initialization, the RS/RA interaction uses the local link address. After the acquisition of the network prefix, the sensor nodes uses the global address to register with the gateway via the NS/NA pair, and then follows the neighbor discovery information maintenance process normally.

The sensor nodes periodically multicast RS. Fig.9 shows the data of printf in the nodes. The gateway unicasts RA to the sensor nodes, which carries two 6CO options: the Ethernet and the 6LoWPAN network prefix, as shown in fig.10.The sensor nodes unicast NS to the gateway, which carries the ARO option as shown in fig.11.The gateway unicasts NA back to the nodes, which carries the ARO option as shown in fig. 12 .

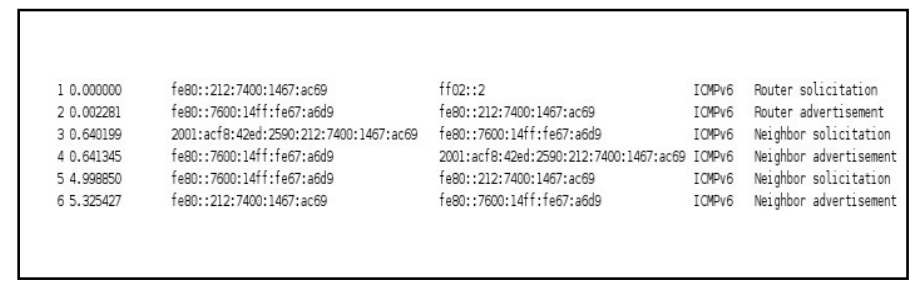

Figure 8 Network initialization

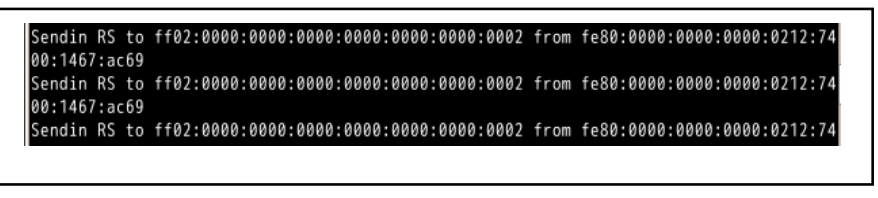

Figure 9 Sensor nodes periodically multicast RS 


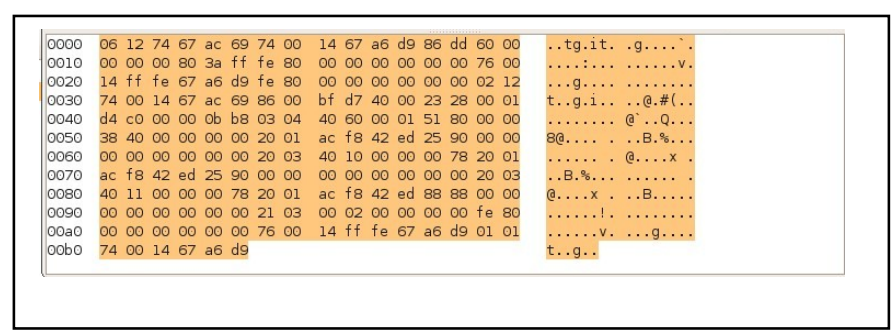

Figure $10 \mathrm{RA}$ message carrying $6 \mathrm{CO}$

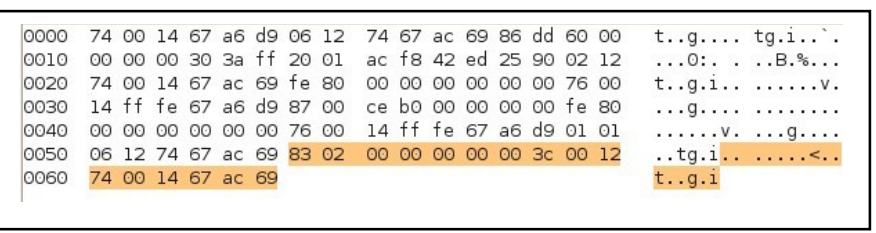

Figure 11 NS message carrying the ARO option

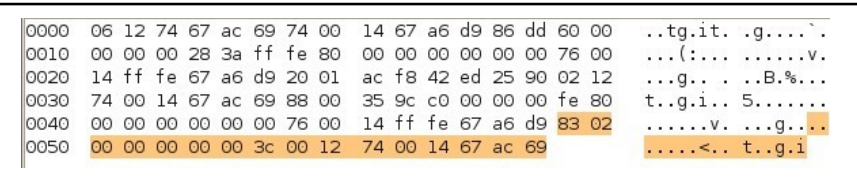

Figure 12 NA message carrying ARO

\begin{tabular}{|c|c|c|c|c|}
\hline 1331.600069 & 2001:act8:42ed:2590:222:7400:14657:c669 & te80: $77600: 144 t:: t e 67: 6069$ & IOPV6 & Neaghbor solucitation \\
\hline 1431.662647 & fe80: $77000: 14 f f:\{e 67: a 6 d 9$ & 2001:act8:4220:2590::212:74000:1467:ac69 & IOPV6 & Neighbor advertisenent \\
\hline 1532.084499 & fe80::212:7400:1467:ac69 & $f \in 80:: 76000: 14 f f: f e 67: a 6 d 9$ & IOPV6 & Neighbor advertisenent \\
\hline 1636.661442 & fe80::7600:14ff:fe67:a6d9 & 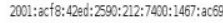 & IOPV6 & Neighbor solicitation \\
\hline 1737.124348 & 2001:acf8: 42ed::2500:212:7400:1067:ac699 & fe80: $77600: 14 f f: f e 67: 06 d 9$ & IOPV6 & Neighbor abvertisenent \\
\hline 1873.765014 & 2001:act8:42ed::2500:212:7400:1457:ac69 & fee80: :7600:14ff:fe67:06d9 & IOPV6 & Neighbor solicitation \\
\hline 1973.786287 & fe80: :7600:14ff:feef7:a6d9 & 2001:acc8:4220: 2590::22:77400:1467:ac69 & IOPV6 & Neighbor advertisenent \\
\hline 20115.718782 & 2001:acf8: $42 \mathrm{ed}: 2590: 221: 7400: 1457: a c 69$ & fe80: :7600: 14f::fe67::60d9 & IOPV6 & Neighbor solicitation \\
\hline 21115.718920 & fe80: :7600:14ff:fe67:a6d9 & 2001:acc8::4200: :390::212:7400:1467:ac69 & IOPV6 & Neighbor advertisenent \\
\hline
\end{tabular}

Figure 13 Periodic maintenance of the address information

After networking, the nodes unicasts NS carrying ARO to the gateway in order to maintain the registration status of the neighbor's cache in the gateway (it takes the node address 60 s to register with the gateway, so the node will send the registration message to the gateway when $1 / 3$ of the registration time is left, i.e. 40 seconds later). After the reception, the gateway will acknowledge by unicasting NA carrying ARO. As shown in the following figure, the message pairs are $\{13,14\},\{18,19\}$ and $\{20,21\}$. In addition, $\{15$, $16\}$ and $\{17,18\}$ are the NS and NA messages generated during neighbor discovery. They do not carry ARO. To achieve forward compatibility, these messages are not banned, as shown in fig. 13.

After networking, the nodes unicast RS to the gateway every 90s in order to update the routing status (the lifetime of each context item is 120 s, and the gateway will ask the gateway for new context status when the lifetime has only 30 s left). After the reception, the gateway will acknowledge by unicasting RA carrying $6 \mathrm{CO}$ and $\mathrm{ABRO}$.

\section{CONCLUSION}

This paper studies the 6LoWPAN-based neighbor discovery protocol and proposes a 6LoWPAN-based basic network architecture. Based on the proposed architecture, we also provide the scheme for configuring the 6LoWPAN node addresses and the basic neighbor interaction protocol. Meanwhile, the context management and distribution scheme for the expanded 6LoWPAN domain is proposed as an approach to the problem of the standard 6Lowpan-ND protocol which does not support the expanded 6lowpan network. Finally,6lowpan networking and neighbor discovery is implemented via simulations to demonstrate the effectiveness of the proposed protocols.

\section{ACKNOWLEDGMENT}

This work is supported by the National Natural Science Foundation of China (60973140, 61170276, 61373135), The research project of Jiangsu Province (BY2013011), The major project of Jiangsu Province University Natural Science (12KJA520003).

\section{REFERENCES}

[1] Kushalnagar, N., G. Montenegro, and C. Schumacher, IPv6 over lowpower wireless personal area networks (6LoWPANs): overview, assumptions, problem statement, and goals. RFC4919, August, 2007. 10.

[2] Group, I.W., Standard for Part 15.4: Wireless Medium Access Control (MAC) and Physical Layer (PHY) Specifications for Low Rate Wireless Personal Area Networks (LR-WPANs). ANSI/IEEE 802.15, 2003. 4.

[3] Ko, J., et al., Connecting low-power and lossy networks to the internet. Communications Magazine, IEEE, 2011. 49(4): p. 96-101.

[4] Seliem, M.A.M., K.M.F. Elsayed, and A. Khattab. Performance evaluation and optimization of neighbor discovery implementation over Contiki OS. in Internet of Things (WF-IoT), 2014 IEEE World Forum on. 2014:119-123.

[5] Montavont, J., C. Cobarzan, and T. Noel. Theoretical analysis of IPv6 stateless address autoconfiguration in Low-power and Lossy Wireless Networks. in Computing \& Communication Technologies - Research, Innovation, and Vision for the Future (RIVF), 2015 IEEE RIVF International Conference on. 2015:198-203.

[6] Shelby, Z., et al., Neighbor Discovery Optimization for IPv6 over LowPower Wireless Personal Area Networks (6LoWPANs). 2012, RFC 6775, November.

[7] Narten, T., et al., Neighbor discovery for IP version 6 (IPv6). 2007. 